\title{
A grading system combining architectural features and mitotic count predicts recurrence in stage I lung adenocarcinoma
}

Kyuichi Kadota ${ }^{1,2}$, Kei Suzuki ${ }^{1}$, Stefan S Kachala ${ }^{1}$, Emily C Zabor ${ }^{3}$, Camelia S Sima ${ }^{3}$, Andre L Moreira ${ }^{4}$, Akihiko Yoshizawa, ${ }^{4,5}$, Gregory J Riely ${ }^{6}$, Valerie W Rusch ${ }^{1}$, Prasad S Adusumilli ${ }^{1,7}$ and William D Travis ${ }^{4}$

${ }^{1}$ Division of Thoracic Service, Department of Surgery, Memorial Sloan-Kettering Cancer Center, New York, NY, USA; ${ }^{2}$ Department of Diagnostic Pathology, Kagawa University, Kagawa, Japan; ${ }^{3}$ Department of Epidemiology and Biostatistics, Memorial Sloan-Kettering Cancer Center, New York, NY, USA; ${ }^{4}$ Department of Pathology, Memorial Sloan-Kettering Cancer Center, New York, NY, USA; ${ }^{5}$ Department of Laboratory Medicine, Shinshu University Hospital, Matsumoto, Japan; ${ }^{6}$ Thoracic Oncology Services, Division of Solid Tumor Oncology, Department of Medicine, Memorial Sloan-Kettering Cancer Center, New York, NY, USA and ${ }^{7}$ Center for Cell Engineering, Memorial Sloan-Kettering Cancer Center, New York, NY, USA

The International Association for the Study of Lung Cancer (IASLC)/American Thoracic Society (ATS)/European Respiratory Society (ERS) has recently proposed a new lung adenocarcinoma classification. We investigated whether nuclear features can stratify prognostic subsets. Slides of $\mathbf{4 8 5}$ stage I lung adenocarcinoma patients were reviewed. We evaluated nuclear diameter, nuclear atypia, nuclear/cytoplasmic ratio, chromatin pattern, prominence of nucleoli, intranuclear inclusions, mitotic count/10 high-power fields (HPFs) or $2.4 \mathrm{~mm}^{2}$, and atypical mitoses. Tumors were classified into histologic subtypes according to the IASLC/ATS/ERS classification and grouped by architectural grade into low (adenocarcinoma in situ, minimally invasive adenocarcinoma, or lepidic predominant), intermediate (papillary or acinar), and high (micropapillary or solid). Log-rank tests and Cox regression models evaluated the ability of clinicopathologic factors to predict recurrence-free probability. In univariate analyses, nuclear diameter $(P=0.007)$, nuclear atypia $(P=0.006)$, mitotic count $(P<0.001)$, and atypical mitoses $(P<0.001)$ were significant predictors of recurrence. The recurrence-free probability of patients with high mitotic count $(\geq 5 / 10 \mathrm{HPF}: n=175)$ was the lowest $(5$-year recurrence-free probability $=73 \%$ ), followed by intermediate (2-4/10 HPF: $n=106,80 \%)$, and low (0-1/10 HPF: $n=204,91 \%, P<0.001)$. Combined architectural/ mitotic grading system stratified patient outcomes $(P<0.001)$ : low grade (low architectural grade with any mitotic count and intermediate architectural grade with low mitotic count: $n=201,5$-year recurrence-free probability $=92 \%$ ), intermediate grade (intermediate architectural grade with intermediate-high mitotic counts: $n=206,78 \%$ ), and high grade (high architectural grade with any mitotic count: $n=78,68 \%$ ). The advantage of adding mitotic count to architectural grade is in stratifying patients with intermediate architectural grade into two prognostically distinct categories $(P=0.001)$. After adjusting for clinicopathologic factors including sex, stage, pleural/lymphovascular invasion, and necrosis, mitotic count was not an independent predictor of recurrence $(P=0.178)$. However, patients with the high architectural/mitotic grade remained at significantly increased risk of recurrence (high vs low: $P=0.005$ ) after adjusting for clinical factors. We proposed this combined architectural/ mitotic grade for lung adenocarcinoma as a practical method that can be applied in routine practice.

Modern Pathology (2012) 25, 1117-1127; doi:10.1038/modpathol.2012.58; published online 13 April 2012

Keywords: architectural grade; histologic subtype; lung adenocarcinoma; mitosis; recurrence

Correspondence: Dr WD Travis, MD, Department of Pathology, Memorial Sloan-Kettering Cancer Center, 1275 York Avenue, Room C563E, New York, NY 10065, USA.

E-mail: travisw@mskcc.org

Received 15 December 2011; revised 9 February 2012; accepted 9 February 2012; published online 13 April 2012
Adenocarcinoma is the most frequent histologic type of lung cancer in most countries. ${ }^{1}$ Increasing evidence suggests that morphologic characteristics, such as architectural and nuclear features, of lung 
adenocarcinoma can stratify tumors with respect to prognosis. $^{2-10}$ The newly proposed International Association for the Study of Lung Cancer (IASLC)/ American Thoracic Society (ATS)/European Respiratory Society (ERS) International Multidisciplinary Classification of Lung Adenocarcinoma clearly emphasizes the prognostic significance of histologic subtypes in lung adenocarcinoma, ${ }^{11}$ a finding that has been validated in independent data sets. ${ }^{12,13}$

The utility of a grading system based on nuclear features has already been established in other carcinomas, such as breast, ${ }^{14,15}$ kidney, ${ }^{16}$ and bladder. ${ }^{17,18}$ Although previous studies have demonstrated the prognostic value of nuclear features in lung adenocarcinoma, ${ }^{5,7}$ a method incorporating architectural and nuclear features into a clinically prognostic grading system has not been established in lung adenocarcinoma. We herein report our findings of nuclear features in predicting recurrence based on comprehensive pathologic review of 485 patients diagnosed with stage I lung adenocarcinoma. The aim of this study is to develop a grading system based on architectural (histologic subtyping) and nuclear features that can predict recurrence in stage I lung adenocarcinoma patients. Our goal is to identify patients with early stage lung adenocarcinoma who are at high risk for recurrence in order to improve classification of patients for future clinical trials.

\section{Materials and methods}

\section{Patients}

Clinical data for 485 patients diagnosed with pathologic stage I lung adenocarcinoma between 1995 and 2005 at Memorial Sloan-Kettering Cancer Center was collected through the Division of Thoracic Service, Department of Surgery and Pathology database. Internal review board approval was obtained for this study. Variables recorded in the prospectively maintained database included age, gender, smoking history, laterality of disease, surgical procedure, and tumor-nodal-metastasis (TNM) stage. Patients were staged according to the seventh edition of the American Joint Committee on Cancer TNM Staging Manual for lung cancers. ${ }^{19}$

The cases in this study were included in a previous report by Yoshizawa et $a l^{12}$ to validate the newly proposed IASLC/ATS/ERS Lung Adenocarcinoma Classification $(n=514)$, and a subset of these cases was previously reported by Sica et $a l^{4}$ to propose a grading system based on tumor architecture $(n=366)$. In the current study, 12 tumors of invasive mucinous adenocarcinoma and 9 tumors of colloid adenocarcinoma were excluded from the original data set because both subtypes had distinctly different morphology, in particular nuclear characteristics, and worse prognosis; ${ }^{11,12}$ so this study is focused on nonmucinous adenocarcinomas. In invasive mucinous adenocarcinoma, nuclear atypia is usually inconspicuous or absent. Colloid adeno- carcinoma shows abundant extracellular mucin pools, in which tumor cells may be inconspicuous. ${ }^{11,12}$

\section{Histologic Evaluation}

All available hematoxylin and eosin (H\&E)-stained slides of all lung adenocarcinoma patients were reviewed by a pathologist (KK), and problem cases were reviewed by two pathologists (KK and WDT). For each case, we evaluated (1) nuclear diameter, (2) nuclear atypia, (3) nuclear/cytoplasmic (N/C) ratio, (4) chromatin pattern, (5) prominence of nucleoli, (6) intranuclear inclusions, (7) mitotic count, and (8) atypical mitoses.

Nuclear features were evaluated using high-power field $(\mathrm{HPF})$ at $\times 400$ magnification $\left(0.237 \mathrm{~mm}^{2}\right.$ field of view) using an Olympus BX51 microscope (Olympus, Tokyo, Japan) with a standard eyepiece of $22 \mathrm{~mm}$ diameter. All nuclear features were assessed in the region of the tumor with the greatest abnormal features after scanning through the entire set of tumor slides at intermediate-power $(\times 100)$ magnification. Mitoses were evaluated in the $50 \mathrm{HPF}$ areas with the highest mitotic activity ${ }^{5,20-22}$ and counted as an average of mitotic figures per $10 \mathrm{HPF}$ $\left(2.4 \mathrm{~mm}^{2}\right.$ area). ${ }^{21}$

For nuclear diameter, we selected at least $3 \mathrm{HPF}$ with the largest nuclei, and evaluated average nuclear diameter among at least 100 tumor cells using as reference nearby small lymphocytes, which typically measure $\sim 4.0 \mu \mathrm{m},{ }^{9}$ and classified it as follows: small nuclei-nuclear diameter $\leq 3$ small lymphocytes (Figure 1a), intermediate nuclei-3 small lymphocytes <nuclear diameter $\leq 5$ small lymphocytes (Figure 1b), and large nuclei-nuclear diameter $>5$ small lymphocytes (Figure 1c)..$^{8,9}$ Nuclear atypia was recorded in the area of tumor with the highest degree of atypia and $>5 \%$ of the entire tumor area needed to be affected. The degree of atypia was assessed as follows: mild atypiauniform nuclei in size and shape (Figure 1a), moderate atypia-nuclei in intermediate size with slight irregularity in shape (Figure 1b), and severe atypia-enlarged nuclei of varied sizes and irregular contours, with some nuclei at least twice as large as others (Figure 1d). ${ }^{5-7,15,17} \mathrm{~N} / \mathrm{C}$ ratio was graded as the following three categories: low $\mathrm{N} / \mathrm{C}$ ratio- $<1 / 3$ nucleus to cytoplasm area (Figure 1b), intermediate $-1 / 3-2 / 3$ (Figure 1a), and high $->2 / 3$ (Figure 1e). Chromatin pattern was graded as the following three categories: homogeneous (Figure 1e), finely granular (Figure 1a), and coarsely granular (Figure 1d). ${ }^{3,23}$ Prominence of nucleoli was graded as the following three categories: indistinct-inconspicuous at intermediate-power $(\times 100)$ magnification (Figure 1e), distinct-conspicuous at intermediatepower magnification (Figure 1a), and large-larger than the size of a small lymphocyte (Figure 1d). Intranuclear inclusions were determined as present or absent in an examination of $50 \mathrm{HPF}$ (Figure 1f). ${ }^{24}$ 

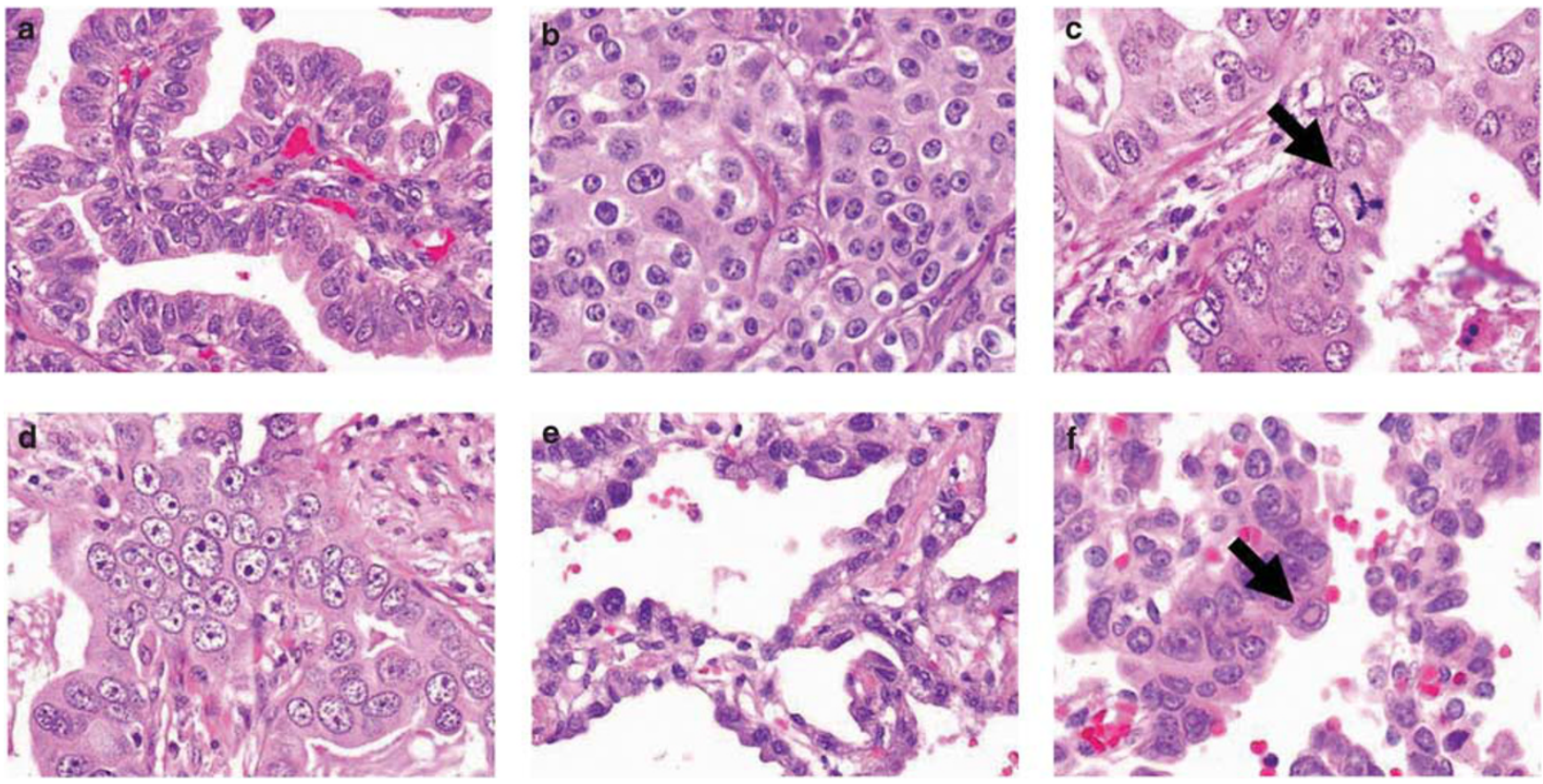

Figure 1 Nuclear features of lung adenocarcinoma (hematoxylin and eosin stain; original magnification, $\times 400$ : $(\mathbf{a}-\mathbf{e})$, $\times 600$ : f). (a) Tumor cells showing mild nuclear atypia with small nuclei, intermediate nuclear/cytoplasmic (N/C) ratio, fine granular chromatin, and distinct nucleoli. (b) Tumor cells showing moderate nuclear atypia with intermediate size nuclei, and low N/C ratio. (c) Tumor cells showing large nuclei with atypical mitosis (arrow). (d) Tumor cells showing severe nuclear atypia with coarse granular chromatin, and large nucleoli. (e) Tumor cells showing high N/C ratio, homogeneous chromatin, and indistinct nucleoli. (f) Tumor cells with intranuclear inclusion (arrow).

We used the following criteria to distinguish mitotic figures from pyknotic cells: absence of a nuclear membrane or a central clear zone, presence of hairy rather than triangular or spiky projections, and cytoplasmic basophilia rather than eosinophilia. ${ }^{22}$ Areas of necrosis and prominent stromal fibrosis or inflammation were avoided whenever possible. Tumors were graded into the following three groups by mitotic count: low- $0-1 / 10$ $\mathrm{HPF}$, intermediate-2-4/10 HPF, and high $-\geq 5 / 10$ HPF. Atypical mitoses were defined as the presence of abnormal chromosome spread, tripolar or quadripolar forms, circular, or bizarre forms. ${ }^{25}$ Atypical mitoses were considered present if any were observed after examination of $50 \mathrm{HPF}$ (Figure 1c). ${ }^{24}$

Histological subtyping was done according to the IASLC/ATS/ERS classification of lung adenocarcinoma. ${ }^{11}$ Each tumor was previously reviewed using comprehensive histological subtyping, recording the percentage of each histological component in 5\% increments. ${ }^{12}$ Tumors were classified as (1) adenocarcinoma in situ, (2) minimally invasive adenocarcinoma, (3) lepidic predominant, (4) papillary predominant, (5) acinar predominant, (6) micropapillary predominant, and (7) solid predominant. The predominant pattern was defined as the morphological subtype in greatest proportion. In addition, tumors were classified according to the architectural grading system based on histologic subtype. ${ }^{4,12}$ as (1) low grade-adenocarcinoma in situ, minimally invasive adenocarcinoma or lepidic predominant; (2) intermediate grade-papillary or acinar; (3) high grade-micropapillary or solid. Although adenocarcinoma in situ and minimally invasive adenocarcinoma should have favorable prognosis, these cases were included to validate their nuclear characteristics, which have not been reported in detail.

We also investigated several histological features: (1) visceral pleural invasion was classified as absent (PL0) or present (PL1, PL2); ${ }^{19}$ (2) presence of lymphatic and vascular invasion was also noted if at least one tumor cell cluster was visible in a lymphatic vessel, vein, or artery, respectively; and (3) necrosis was classified as present or absent.

\section{Tissue Microarray}

Formalin-fixed, paraffin-embedded tumor specimens were used for tissue microarray construction. Briefly, four representative tumor areas were marked on H\&E-stained slides, and cylindrical $0.6-\mathrm{mm}$ tissue cores were arrayed from the corresponding paraffin blocks into a recipient block using an automated tissue arrayer ATA-27 (Beecher Instruments, Sun Prairie, WI, USA), resulting in seven tissue microarray blocks. From each tissue microarray, $4-\mu \mathrm{m}$-thick paraffin sections were prepared for 
immunohistochemistry. In all, 435 cases with adequate cores were available for immunohistochemical analysis.

\section{Immunohistochemistry and Scoring of Ki-67}

Briefly, 4- $\mu \mathrm{m}$ sections from the tissue microarray blocks were deparaffinized in xylene and dehydrated in graded alcohols. Standard avidin-biotin complex peroxidase technique was used for immunohistochemical stain of anti-Ki-67 antibody (clone MIB-1, Immunotech, Westbrook, ME, USA; diluted at 1:100). Sections were stained using a Ventana Discovery XT automated immunohistochemical stainer (Ventana, Tucson, AZ, USA) according to the manufacturer's guidelines. Diaminobenzidine was used as the chromogen and hematoxylin as the nuclear counterstain. Positive control tissues were stained in parallel with the study cases.

Ki-67 proliferation index was recorded as the percentage of tumor cells with nuclear positive immunostaining in each tissue microarray core. The average percentage of the tumor cores were considered as Ki-67 proliferation index for each patient. Tumors were classified into the following two groups by Ki-67 proliferation index using the mean value: ${ }^{26}$ low $-<10 \%$ and high $-\geq 10 \%$.

\section{Statistical Analysis}

Associations between clinicopathologic variables and histologic and nuclear findings were analyzed using Fisher exact test for categorical variables and Wilcoxon test for continuous variables. The main outcome of interest was recurrence-free probability. Patients were followed up from the time of surgery to documented recurrence, and censored at date of death or last follow-up, if no recurrence had occurred. Previous studies we have published from these cases examined the outcome of disease-free survival, ${ }^{4,12}$ which incorporates both recurrence and death from lung cancer as events. Because establishing cause of death in low-grade tumors can be difficult and subject to uncertainty, here we only focus on recurrence only. Recurrence-free probability was estimated using the Kaplan-Meier method and non-parametric group comparisons were performed using the log-rank test. Multivariate analyses were performed using Cox proportional hazards regression. We first examined the univariate association between each nuclear feature and recurrence-free probability to determine candidate variables for inclusion in a multivariate model. Next, we used a Spearman correlation matrix to examine the correlation between nuclear features. We examined the features significant $(P<0.05)$ on univariate analysis in a multivariate model. Nuclear features that remained significant in multivariate analysis were combined with architectural grade to form a combined architectural/nuclear grading system based on 5-year recurrence-free probability. Clinicopathologic variables were included in multivariate analysis of the architectural/nuclear grading system if they were significantly associated with recurrence-free probability in univariate analysis.

Since bias can be introduced into survival analysis through censoring patients who died, a sensitivity analysis was performed using competing risks. The competing risk of death from any cause did not have a significant association with any nuclear features or architectural grade in univariate analysis. Furthermore, associations between nuclear features, architectural grade, and clinicopathologic variables with recurrence in the presence of death from any cause as a competing risk did not differ from the associations found through survival analysis. Therefore, only the recurrence analysis results are presented here.

All $P$-values were based on two-tailed statistical analysis, and a $P$-value $<0.05$ was considered to indicate statistical significance. Statistical analyses were conducted using SAS version 9.2 (SAS Institute, Cary, NC, USA) and R (R Development Core Team, 2010), including the 'survival' and 'cmprsk' packages.

\section{Results}

\section{Patient Demographics}

Patient demographics are outlined in Table 1. The study cohort consisted of 485 stage I lung adenocarcinoma patients with a median age of 69 years (range: $33-89), 37 \%(n=179)$ of whom were males. A majority of the patients $(71 \% ; n=346)$ were former smokers, with 14\% $(n=67)$ current smokers. The tumor involved the left lung in $42 \%(n=205)$ of the cases. By surgical procedure, 10\% $(n=48)$ underwent wedge resection, $6 \%(n=30)$ underwent segmentectomy, and the remaining $84 \%$ had lobectomy $(n=403)$, bilobectomy $(n=3)$, or pneumonectomy $(n=1)$. The distribution of T stage consisted of $42 \%(n=205)$ T1a, 20\% $(n=95) \mathrm{T} 1 \mathrm{~b}$, and $38 \%$ $(n=185)$ T2. By pathologic stage, there were $62 \%$ $(n=300)$ stage IA patients and $38 \%(n=185)$ stage IB. A minority of tumors had evidence of pleural invasion, with $17 \%$ PL1 $(n=83)$ and $4 \%$ PL2 $(n=18)$. Lymphatic invasion was detected in $29 \%$ $(n=139)$, vascular invasion in $30 \%(n=145)$, and necrosis in $19 \%(n=94)$. The median follow-up period for patients who did not recur was 53.0 months (range: 0.3-160.0). Seventy-eight experienced disease recurrence; 107 patients died during the study time without documented recurrence.

In univariate analysis, male gender $(P=0.001)$, higher stage (stage IB: $P<0.001$ ), pleural invasion $(P=0.008)$, lymphatic invasion $(P<0.001)$, vascular invasion $(P<0.001)$, and necrosis $(P<0.001)$ were associated with lower recurrence-free probability (Table 1). 
Table 1 Patient demographics and its association with mitotic count and recurrence

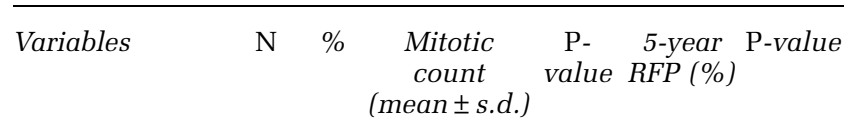

\begin{tabular}{|c|c|c|c|c|c|c|}
\hline $\begin{array}{l}\text { All patients } \\
\text { Age (years) }\end{array}$ & 485 & 100 & $5.1 \pm 6.8$ & 0.002 & & 0.320 \\
\hline$\leq 65$ & 176 & 36 & $4.5 \pm 6.7$ & & 80 & \\
\hline$>65$ & 309 & 64 & $6.1 \pm 6.9$ & & 84 & \\
\hline Gender & & & & 0.355 & & 0.001 \\
\hline Female & 306 & 63 & $4.8 \pm 6.2$ & & 87 & \\
\hline Male & 179 & 37 & $5.7 \pm 7.7$ & & 73 & \\
\hline Smoking status & & & & $<0.001$ & & 0.088 \\
\hline Never & 72 & 15 & $2.1 \pm 2.4$ & & 90 & \\
\hline $\begin{array}{l}\text { Former/ } \\
\text { current }\end{array}$ & 413 & 85 & $5.7 \pm 7.2$ & & 81 & \\
\hline Laterality & & & & 0.274 & & 0.570 \\
\hline Left & 205 & 42 & $5.0 \pm 6.9$ & & 81 & \\
\hline Right & 280 & 58 & $5.2 \pm 6.7$ & & 83 & \\
\hline Stage & & & & $<\mathbf{0 . 0 0 1}$ & & $<0.001$ \\
\hline IA & 300 & 62 & $4.1 \pm 5.6$ & & 87 & \\
\hline IB & 185 & 38 & $6.8 \pm 8.1$ & & 74 & \\
\hline Pleural invasion & & & & $<0.001$ & & 0.008 \\
\hline Absence & 384 & 79 & $4.6 \pm 6.2$ & & 84 & \\
\hline Presence & 101 & 21 & $7.1 \pm 8.3$ & & 75 & \\
\hline $\begin{array}{l}\text { Lymphatic } \\
\text { invasion }\end{array}$ & & & & $<0.001$ & & 0.001 \\
\hline Absence & 346 & 71 & $4.3 \pm 6.0$ & & 86 & \\
\hline Presence & 139 & 29 & $7.1 \pm 8.2$ & & 74 & \\
\hline $\begin{array}{l}\text { Vascular } \\
\text { invasion }\end{array}$ & & & & $<0.001$ & & $<\mathbf{0 . 0 0 1}$ \\
\hline Absence & 340 & 70 & $3.6 \pm 5.1$ & & 86 & \\
\hline Presence & 145 & 30 & $8.7 \pm 8.7$ & & 74 & \\
\hline Necrosis & & & & $<0.001$ & & $<0.001$ \\
\hline Absence & 391 & 81 & $3.5 \pm 5.0$ & & 87 & \\
\hline Presence & 94 & 19 & $11.9 \pm 8.7$ & & 62 & \\
\hline $\begin{array}{l}\text { Histologic } \\
\text { subtype }\end{array}$ & & & & $<\mathbf{0 . 0 0 1}$ & & $<0.001$ \\
\hline AIS/MIA & 9 & 2 & $0.1 \pm 0.3$ & & 100 & \\
\hline Lepidic & 27 & 6 & $1.2 \pm 1.4$ & & 89 & \\
\hline Papillary & 140 & 29 & $3.7 \pm 4.9$ & & 83 & \\
\hline Acinar & 231 & 48 & $4.8 \pm 6.5$ & & 85 & \\
\hline Micropapillary & 12 & 3 & $5.0 \pm 3.4$ & & 64 & \\
\hline Solid & 66 & 14 & $11.6 \pm 9.1$ & & 69 & \\
\hline $\begin{array}{l}\text { Architectural } \\
\text { grade }\end{array}$ & & & & $<\mathbf{0 . 0 0 1}$ & & $<\mathbf{0 . 0 0 1}$ \\
\hline Low & 36 & 7 & $0.9 \pm 1.3$ & & 92 & \\
\hline Intermediate & 371 & 76 & $4.4 \pm 6.0$ & & 84 & \\
\hline High & 78 & 16 & $10.6 \pm 8.8$ & & 68 & \\
\hline
\end{tabular}

Abbreviations: AIS, adenocarcinoma in situ; RFP, recurrence-free probability; MIA, minimally invasive adenocarcinoma.

Significant $P$-values are shown in bold: $P$-values for mitotic count from Wilcoxon test and for survival from log-rank test.

\section{Association Between Nuclear Features and Recurrence}

Among the nuclear features examined, nuclear diameter $(P=0.007)$, nuclear atypia $(P=0.006)$, mitotic count $(P<0.001)$, and atypical mitoses
Table 2 Association between nuclear features and recurrence

\begin{tabular}{|c|c|c|c|c|}
\hline Variables & $\mathrm{N}$ & $\%$ & 5-year RFP (\%) & $\mathrm{P}$-value \\
\hline Nuclear diameter & & & & 0.007 \\
\hline Small & 232 & 48 & 86 & \\
\hline Intermediate & 150 & 31 & 82 & \\
\hline Large & 103 & 21 & 74 & \\
\hline Nuclear atypia & & & & 0.006 \\
\hline Mild & 231 & 48 & 87 & \\
\hline Moderate & 139 & 29 & 81 & \\
\hline Severe & 115 & 24 & 75 & \\
\hline Nuclear/cytoplasmic ratio & & & & 0.068 \\
\hline Low/intermediate & 391 & 81 & 80 & \\
\hline High & 94 & 19 & 91 & \\
\hline Chromatin pattern & & & & 0.092 \\
\hline Homogeneous & 85 & 18 & 87 & \\
\hline Fine granular & 229 & 47 & 83 & \\
\hline Coarse granular & 171 & 35 & 79 & \\
\hline Prominence of nucleoli & & & & 0.059 \\
\hline Indistinct & 133 & 27 & 87 & \\
\hline Distinct & 217 & 45 & 82 & \\
\hline Large & 135 & 28 & 87 & \\
\hline Intranuclear inclusion & & & & 0.190 \\
\hline Absence & 441 & 91 & 82 & \\
\hline Presence & 44 & 9 & 88 & \\
\hline Mitotic count & & & & $<\mathbf{0 . 0 0 1}$ \\
\hline Low & 204 & 42 & 91 & \\
\hline Intermediate & 106 & 22 & 80 & \\
\hline High & 175 & 36 & 73 & \\
\hline Atypical mitoses & & & & $<0.001$ \\
\hline Absence & 362 & 75 & 86 & \\
\hline Presence & 123 & 25 & 72 & \\
\hline
\end{tabular}

Abbreviation: RFP, recurrence-free probability.

Significant $P$-values are shown in bold: all $P$-values from log-rank test.

$(P<0.001)$ were shown to be significantly associated with recurrence-free probability in univariate analyses (Table 2). The recurrence-free probability of patients with large nuclear diameter $(n=103)$ was the lowest (5-year recurrence-free probability $=74 \%)$, followed by intermediate $(n=150$, $82 \%)$, and small $(n=232,86 \%, P=0.007)$. The recurrence-free probability of patients with severe atypia $(n=115)$ was the lowest (5-year recurrencefree probability $=75 \%$ ), followed by moderate $(n=139,81 \%)$, and mild $(n=231,87 \%, P=0.006)$. Mitotic count ranged from 0 to 43 per $10 \mathrm{HPF}$ (median $=2.0$; mean \pm s.d. $=5.1 \pm 6.8$ ). The recurrence-free probability of patients with high mitotic count $(n=175)$ was the lowest (5-year recurrencefree probability $=73 \%)$, followed by intermediate $(n=106,80 \%)$, and low $(n=204,91 \%, P<0.001)$ (Figure 2a). The presence of atypical mitoses $(n=123)$ was associated with lower 5-year recurrence-free probability $(72 \%)$ compared with their absence $(n=362,86 \%, P<0.001)$. There was a trend for an association between recurrence-free probability and N/C ratio $(P=0.068)$, chromatin pattern $(P=0.092)$, and prominence of nucleoli $(P=0.059)$. 

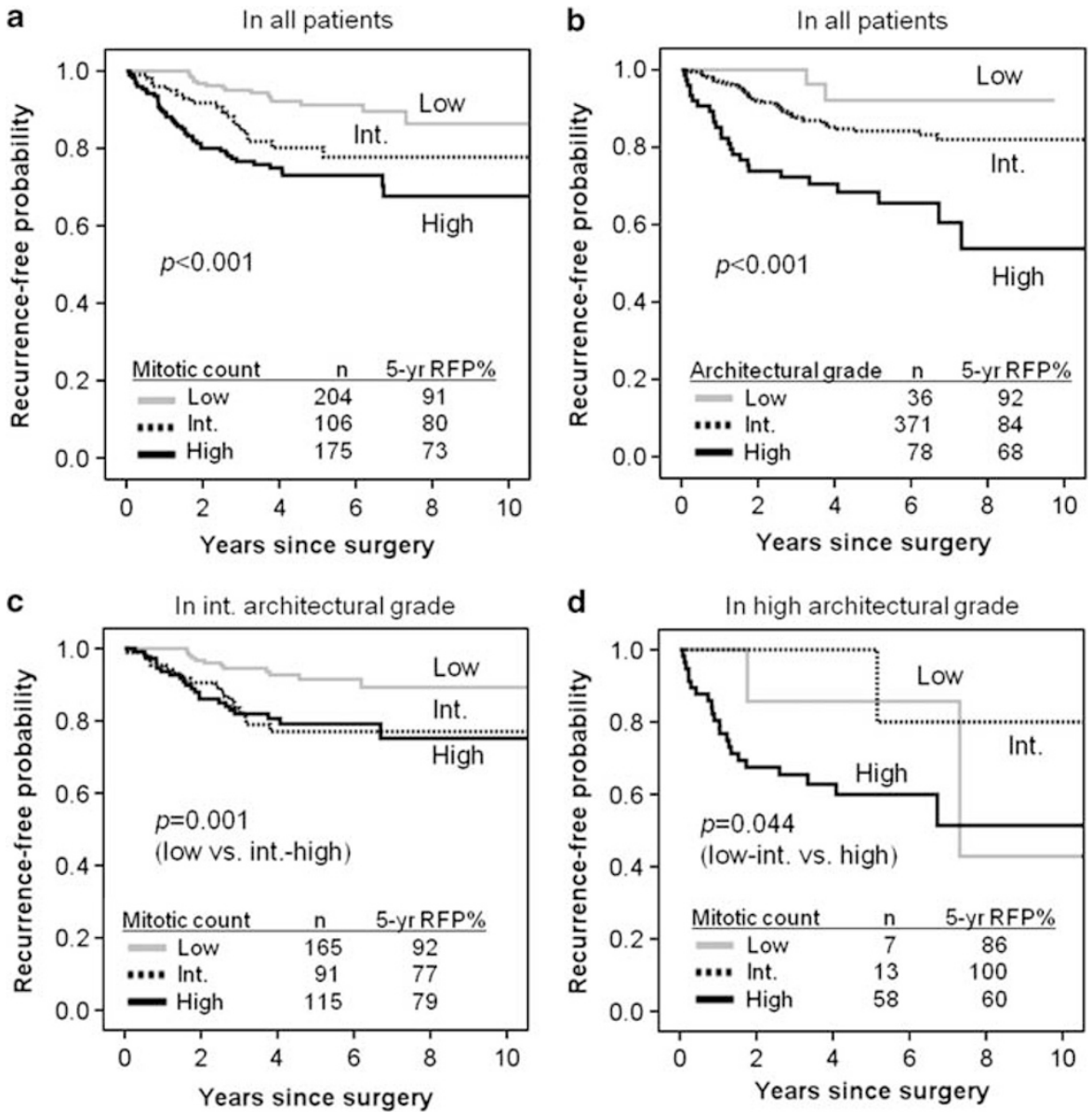

Figure 2 Recurrence-free probability (RFP) by mitotic count and architectural grade. (a) The recurrence-free probability of patients with high mitotic count $(n=175)$ was the lowest ( 5 -year recurrence-free probability $=73 \%)$, followed by intermediate $(n=106,80 \%)$, and low $(n=204,91 \%)$. (b) The RFP of patients with high architectural grade $(n=78)$ was the lowest $(68 \%)$, followed by intermediate $(n=371$, $84 \%)$, and low $(n=36,92 \%)$. (c) Within the intermediate architectural grade, patients with low mitotic count $(n=165)$ had higher 5 -year RFP (92\%) compared with the intermediate $(n=91,77 \%)$-high mitotic count $(n=115,79 \%)$. (d) Within the high architectural grade, patients with high mitotic count $(n=58)$ had lower 5-year RFP $(60 \%)$ compared with the low $(n=7$, $86 \%)$-intermediate mitotic count $(n=13,100 \%)$.

Intranuclear inclusions were not associated with recurrence-free probability.

Among the nuclear features, significant positive correlations were identified between: (1) nuclear diameter and nuclear atypia $(P<0.001),(2)$ prominence of nucleoli and chromatic pattern $(P<0.001)$, and (3) mitotic count and atypical mitoses $(P<0.001)$. In a multivariate analysis including nuclear diameter, prominence of nucleoli, and mitotic count, only mitotic count had a significant independent association with risk of recurrence (high vs low: hazard ratio (HR) $=3.77,95 \%$ confidence interval $(\mathrm{CI})=1.91-7.44, P<0.001$, intermediate vs low: $\mathrm{HR}=2.55,95 \% \mathrm{CI}=1.24-5.26$, $P=0.010$ ). Therefore, all subsequent analyses focused on the role of mitotic count.

Higher mitotic count was significantly associated with older age ( $>65$ years: $P=0.002$ ), smoking history $(P<0.001)$, higher stage (stage IB: $P<0.001)$, pleural invasion $(P<0.001)$, lymphatic invasion $(P<0.001)$, vascular invasion $(P<0.001)$, and necrosis $(P<0.001$; Table 1$)$.

\section{Association Between Histologic Subtypes and Recurrence}

The histologic subtypes and their association with recurrence-free probability are shown in Table 1. By the IASLC/ATS/ERS classification, patients with adenocarcinoma in situ $(n=1)$ or minimally invasive adenocarcinoma $(n=8)$ experienced the highest recurrence-free probability with $100 \%$ at 5 years, followed by lepidic predominant tumors $(n=27$, 5 -year recurrence-free probability $=89 \%$ ), papillary $(n=140,83 \%)$, acinar $(n=231,85 \%)$, micropapillary $(n=12,64 \%)$, and solid predominant adenocarcinomas $(n=66,69 \%)$. The architectural grading system based on histologic subtype stratifies patients into high grade (micropapillary or solid: 
5-year recurrence-free probability $=68 \%$ ), intermediate grade (papillary or acinar: 84\%), and low grade (adenocarcinoma in situ, minimally invasive adenocarcinoma or lepidic predominant: $92 \%$, $P<0.001$; Figure 2b).

\section{Association Between Architectural (Histologic Subtype) and Nuclear (Mitotic Count) Grade}

In $352(73 \%)$ of all tumors, nuclear features with the greatest abnormality and mitotic count with the highest mitotic activity were mainly evaluated in the area of the first predominant histologic pattern: 8 in lepidic, 91 in papillary, 175 in acinar, 12 in micropapillary, and 66 in solid pattern. In 98 (20\%) tumors, nuclear features and mitotic count were evaluated in the area of the second predominant histologic pattern: 10 in papillary, 41 in acinar, 6 in micropapillary, and 41 in solid pattern.

Higher mitotic count was significantly associated with histologic subtype $(P<0.001)$ and architectural grade $(P<0.001$; Table 1$)$. Among tumors with low architectural grade $(n=36), 32(89 \%)$ had low mitotic counts. In contrast, among tumors with high architectural grade $(n=78), 58(74 \%)$ had high mitotic count. Among tumors with intermediate architectural grade $(n=371)$, there were $165(45 \%)$ tumors with low mitotic count, 91 (25\%) with intermediate mitotic count, and 115 (31\%) with high mitotic count. Among tumors with intermediate architectural grade and high mitotic count $(n=115), 42(37 \%)$ had high architectural grade histology as a second predominant pattern. In contrast, among tumors with intermediate architectural grade and low mitotic count $(n=165)$, only 18 $(11 \%)$ had high architectural grade histology as the second predominant pattern.

\section{Combined Architectural/Mitotic Grading System Based on a Combination of Architectural (Histologic Subtype) and Nuclear (Mitotic Count) Grading}

We next investigated whether mitotic count can further stratify patient outcome within each grade based on histologic subtype. Within the low-grade histologic subtype, there was no significant difference of recurrence-free probability between low mitotic count $(n=32, \quad 5$-year recurrence-free probability $=91 \%$ ) and the intermediate-high mitotic count combined group $(n=4,100 \%, P=0.569)$. Within the intermediate architectural grade, patients with low mitotic count $(n=165)$ had higher 5-year recurrence-free probability (92\%) compared with the intermediate $(n=91,77 \%)$-high mitotic count ( $n=115,79 \%, P=0.001$ ) (Figure 2c). Within the high architectural grade, patients with high mitotic count $(n=58)$ had lower 5-year recurrence-free probability $(60 \%)$ compared with the low $(n=7$, $86 \%)$-intermediate mitotic count $(n=13,100 \%$, $P=0.044$; Figure 2d). Despite the high recurrence-

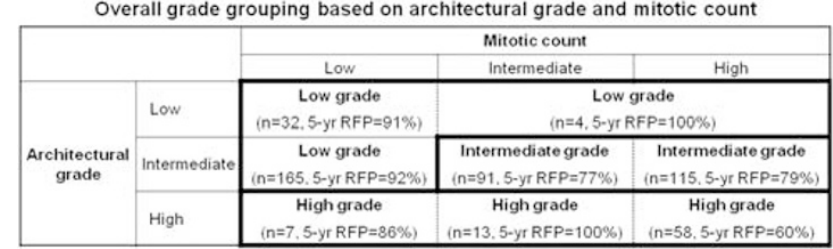

Figure 3 Overall grade grouping based on recurrence-free probability (RFP) by architectural grade and mitotic count. Low architectural/mitotic grade: low architectural grade with any mitotic count (5-year recurrence-free probability $=91-100 \%$ ) and intermediate architectural grade with low mitotic count $(92 \%)$. Intermediate architectural/mitotic grade: intermediate architectural grade with intermediate-high mitotic count (77-79\%). High architectural/mitotic grade: high architectural grade with any mitotic count $(60-100 \%)$.

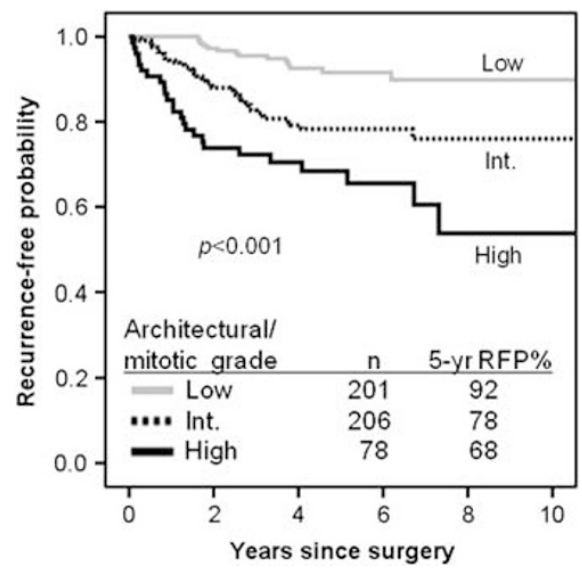

Figure 4 Recurrence-free probability (RFP) by architectural/ mitotic grading system. Patients with the high architectural/ mitotic grade $(n=78)$ had the lowest 5-year RFP $(68 \%)$, followed by intermediate $(n=206,78 \%)$ and low $(n=201,92 \%)$.

free probability for patients with high architectural grade with low/intermediate mitotic count, the small sample size may result in unstable estimates and these patients are likely more similar to other high-grade patients with regard to recurrence.

From these observations, we constructed a combined architectural/mitotic grading system that classifies patients with regard to recurrence risk as (1) low architectural/mitotic grade that consists of low architectural grade with any mitotic count (5year recurrence-free probability $=91-100 \%$ ) and intermediate architectural grade with low mitotic count (92\%), (2) intermediate architectural/mitotic grade that consists of intermediate architectural grade with intermediate-high mitotic count (77$79 \%$ ), and (3) high architectural/mitotic grade that consists of high architectural grade with any mitotic count $(60-100 \%)$ (Figure 3). Patients in the high architectural/mitotic grade $(n=78)$ had the lowest 5 -year recurrence-free probability (68\%), followed by intermediate $(n=206,78 \%)$ and low $(n=201$, $92 \%, P<0.001$; Figure 4). 
Table 3 Results of multivariate Cox proportional hazards model

\begin{tabular}{lccc}
\hline Variable & $H R$ & $95 \%$ CI & P-value \\
\hline Sex (male vs female) & 1.98 & $1.26-3.09$ & $\mathbf{0 . 0 0 3}$ \\
Stage (IB vs IA) & 1.58 & $0.96-2.60$ & 0.072 \\
Pleural invasion & 1.31 & $0.77-2.23$ & 0.325 \\
Lymphatic invasion & 1.21 & $0.72-2.03$ & 0.475 \\
Vascular invasion & 1.04 & $0.62-1.75$ & 0.878 \\
Necrosis & 2.34 & $1.41-3.90$ & $\mathbf{0 . 0 0 1}$ \\
Architectural/mitotic grade & & & \\
$\quad$ Intermediate vs low & 1.94 & $0.99-3.81$ & 0.053 \\
$\quad$ High vs low & 2.96 & $1.39-6.28$ & $\mathbf{0 . 0 0 5}$ \\
\hline
\end{tabular}

Abbreviations: CI, confidence interval; HR, hazard ratio.

Significant $P$-values are shown in bold.

\section{Multivariate Analysis Adjusting for Clinicopathologic} Factors

After adjusting for clinicopathologic factors including sex, stage, pleural/lymphovascular invasion, and necrosis, mitotic count was not an independent predictor of recurrence $(P=0.178)$. However, patients with the high architectural/mitotic grade remained at significantly increased risk of recurrence (high vs low: $P=0.005$ ) after adjusting for clinical factors. In addition, male gender $(P=0.003)$ and necrosis $(P=0.001)$ were independent predictors of decreased recurrence-free probability in the multivariate model (Table 3). Furthermore, architectural/ mitotic grade remained an independent predictor of recurrence even after excluding adenocarcinoma in situ and minimally invasive adenocarcinoma cases $(P=0.024)$.

\section{Association Between Ki-67 Proliferation Index and Recurrence, Mitotic Count, and Histologic Subtype}

Ki-67 proliferation index ranged from 0 to $78 \%$ (median $=5$, mean \pm s.d. $=10 \pm 14$ ). Patients with high Ki-67 proliferation index $(n=141)$ had significantly lower recurrence-free probability (5-year recurrence-free probability $=72 \%$ ) than those with low Ki-67 proliferation index $(n=294,88 \%$, $P<0.001)$. When looking at Ki-67 proliferation index and mitotic count as a continuous variable, a moderately significant positive correlation was found $(r=0.581, P<0.001)$. Tumors with high mitotic count $(n=157)$ had the highest Ki-67 proliferation index (mean \pm s.d. $=19.5 \pm 18.2$ ), followed by intermediate $(n=95,7.9 \pm 7.6)$, and low $(n=183, \quad 3.7 \pm 3.8, \quad P<0.001)$. Tumors with high architectural grade $(n=68)$ had the highest Ki-67 proliferation index (mean \pm s.d. $=20.2 \pm 19.5$ ), followed by intermediate $(n=334,9.0 \pm 11.8)$, and low $(n=33,2.9 \pm 2.7, P<0.001)$.

When Ki-67 proliferation index replaced mitotic count in the combined grade, combined architectural/Ki-67 grade with the intermediate histology separated by high/low Ki-67 proliferation index had a tendency to correlate with increased risk of recurrence in multivariate analysis adjusting for clinicopathologic factors $(P=0.068)$.

\section{Discussion}

In this study, we have demonstrated that mitotic count is a prognostic factor in stage I lung adenocarcinoma and it further stratifies patients with intermediate architectural grade tumors with respect to their risk of recurrence. In addition, our proposed combined architectural/mitotic grading system is an independent predictor of recurrence after adjusting for clinicopathologic factors. The architectural grading comes directly from the histologic subtyping according to the 2011 IASLC/ATS/ERS Lung Adenocarcinoma Classification according to the single predominant pattern. . $^{411,12}$

Compared with other nuclear features we examined, mitotic count was the single most important predictor of recurrence in stage I lung adenocarcinoma patients. This result is consistent with the importance of mitotic count in prior studies of lung cancer including neuroendocrine tumors, ${ }^{6,7,10,21}$ as well as carcinomas of the breast, ${ }^{14,15,20}$ and bladder, ${ }^{18}$ in addition to soft tissue sarcomas. ${ }^{27,28}$ In one study, mitotic counts were not found to be predictive of survival in lung adenocarcinoma. ${ }^{5}$ This may be due to different methods of interpretation or the higher thresholds that were analyzed to stratify the mitotic counts. More importantly, mitotic count was able to identify subsets of intermediate architectural grade that were at favorable vs unfavorable risk of recurrence. This is of notable importance as the intermediate grade histologic subtype, the most problematic in terms of treatment management, comprises $76 \%$ of the cases in our series. A similar high percentage $(55 \%)$ of the intermediate architectural grade adenocarcinomas was recently reported by Yoshizawa et $a .^{13}$ While histologic subtyping alone yielded three significant prognostic architectural grades (low, intermediate, and high with 5-year recurrence-free probability $92 \%, 84 \%$, and $68 \%$, respectively), there is a need to further stratify prognostic subsets of adenocarcinomas with an intermediate architectural grade. By comparison, our proposed combined architectural/mitotic grading system, which is based on a combination of architectural (histologic subtype) and nuclear (mitotic count) grading, yielded three groups of low $(n=201)$, intermediate $(n=206)$, and high $(n=78)$ grade patients with similarly distinct 5 -year recurrence-free probability $(92 \%, 78 \%$, and $68 \%$, respectively).

Proliferation index using anti-Ki-67 antibody has also been used to predict outcome of lung adenocarcinoma patients. ${ }^{26,29,30}$ It has also been correlated with tumor differentiation of lung adenocarcinomas, ${ }^{31}$ but not in the context of precise criteria for architectural grading or in comparison with mitotic counts where they have been demonstrated in 
combination to be an independent predictor of prognosis. Our finding that the Ki-67 proliferation index correlated with mitotic counts as well as recurrence is supportive of the importance of mitotic counts as a measure of adenocarcinoma aggressiveness. It also confirms previously reported associations between Ki-67 proliferation index, mitotic count, and prognosis in lung cancer. ${ }^{29,30,32}$ However, an advantage of the mitotic count is that it can be determined using $\mathrm{H} \& \mathrm{E}$ slides within routine clinical work without the need for immunohistochemistry.

Nuclear grade based on nuclear atypia or mitotic count has been associated with TNM stage and aggressive tumor features such as invasiveness in lung, ${ }^{9,23}$ breast, ${ }^{33}$ and bladder cancer. ${ }^{18}$ These associations are consistent with our results, where mitotic count was significantly associated with older age, smoking history, higher stage, pleural invasion, lymphatic invasion, vascular invasion, necrosis, and higher architectural grade. These observations reflect aggressive tumor biology of lung adenocarcinoma with higher mitotic counts. However, mitotic counts have not been examined in lung adenocarcinomas graded with specific architectural criteria as derived from the histologic subtypes of the IASLC/ATS/ERS adenocarcinoma classification. ${ }^{11,12}$

In addition to mitotic count, we examined other nuclear features and observed that greater nuclear diameter, more severe nuclear atypia, and atypical mitoses were associated with lower recurrence-free probability. While coarse chromatin pattern and prominence of nuclei have been considered as criterion for nuclear atypia in addition to nuclear size and irregularity, ${ }^{5,16,17,33}$ there was only a trend for an association between recurrence-free probability and chromatin pattern or prominence of nuclei in our study.

A criticism of the evaluation of nuclear features is that there can be significant interobserver variability regarding nuclear atypia, ${ }^{14}$ mitoses,${ }^{34}$ and atypical mitoses. ${ }^{35}$ Therefore, standardized and robust definitions, which we have rigorously applied in this study, are necessary if prognostic significance is accorded to the findings of those factors. Another important factor is careful review of all tumor slides at an intermediate magnification $(\times 100)$ to identify the areas with highest nuclear grade. To minimize interobserver variability, Nakazato et $a l^{9}$ and Petersen et $a l^{8}$ recommended that the size of intermingled small lymphocytes be used to evaluate nuclear area and nuclear diameter as an internal control. The criteria for mitoses and atypical mitoses were clearly described in previous reports, ${ }^{22,25}$ which were noted in the methods of our study. In some studies, nuclear morphometry, such as nuclear diameter, nuclear area, and nuclear irregularity, has been evaluated using analytical computer software. ${ }^{9,36}$ Although digital assessment of nuclear morphometry allows for a more objective evaluation of nuclear atypia, such an application is not practical for routine pathologic examination.
In recent years, several attempts have been made to develop an improved histologic classification in lung adenocarcinoma to improve therapeutic decision making beyond current practice standards and further stratify patients for clinical trials. ${ }^{2-11}$ There have also been several reports suggesting that molecular features are prognostic.37,38 However, most of the molecular studies require special techniques and increase costs and the prognostic significance of molecular markers has not been compared with the outcome predicted by the newly proposed adenocarcinoma histologic classification. Our proposed approach by review H\&E-stained slides can be performed with standard histopathologic techniques and incorporated into routine pathologic diagnosis of resected lung adenocarcinoma. This study's comprehensive analysis of nuclear features of lung adenocarcinoma suggests that mitotic count is the most important nuclear feature that predicts recurrence. We propose a combined grading system based on architectural grade (histologic subtype) and mitotic count that efficiently stratifies stage I lung adenocarcinoma patients for their risk of recurrence. Most pathologists are familiar with grading systems based on nuclear features such as nuclear atypia and mitotic count, since similar methods are applied to carcinomas in other organs, such as breast, ${ }^{14,15}$ kidney, ${ }^{16}$ and bladder. ${ }^{17,18}$ The identification of those patients with stage I lung adenocarcinoma who are at high risk for recurrence provides important information for clinical management. While several previous clinical trials applying adjuvant chemotherapy to stage I NSCLC patients found no benefit from treatment, ${ }^{39,40}$ the use of adjuvant chemotherapy for those stage I lung adenocarcinoma patients at high risk of recurrence, as identified by our combined architectural/mitotic grading system, could improve their survival. Translation of our proposed system is practical and can be readily applied in routine practice without the need for special studies. Therefore, it would be of great interest for this proposal to be tested further in future clinical trials to stratify stage I adenocarcinoma patients for adjuvant therapy.

\section{Acknowledgement}

We thank Joe Dycoco for his help with the Lung Adenocarcinoma database within the Division of Thoracic Service, Department of Surgery, Avani Giri and Louie Lopez (Pathology Core Facility) for their help in making tissue microarray, and Irina Linkov (Pathology Core Facility) for her technical assistance in immunohistochemistry. This work was supported, in part, by the International Association for the Study of Lung Cancer (IASLC)-Young Investigator Award; National Lung Cancer Partnership/LUNGevity Foundation Research Grant; Stony Wold-Herbert Fund; American Association for Thoracic Surgery 
(AATS)-Third Edward D Churchill Research Scholarship; Mesothelioma Applied Research Foundation (MARF) Grant in memory of Lance S Ruble; William H Goodwin, and Alice Goodwin the Commonwealth Foundation for Cancer Research and the Experimental Therapeutics Center; New York State Empire Clinical Research Investigator Program (ECRIP); the National Cancer Institute (Grants U54CA137788, U54CA132378); and the US Department of Defense (Grant PR101053).

\section{Disclosure/conflict of interest}

The authors declare no conflict of interest.

\section{References}

1 Devesa SS, Bray F, Vizcaino AP, et al. International lung cancer trends by histologic type: male:female differences diminishing and adenocarcinoma rates rising. Int J Cancer 2005;117:294-299.

2 Motoi N, Szoke J, Riely GJ, et al. Lung adenocarcinoma: modification of the 2004 WHO mixed subtype to include the major histologic subtype suggests correlations between papillary and micropapillary adenocarcinoma subtypes, EGFR mutations and gene expression analysis. Am J Surg Pathol 2008;32:810-827.

3 Okudela K, Woo T, Mitsui H, et al. Morphometric profiling of lung cancers. Its association with clinicopathologic, biologic, and molecular genetic features. Am J Surg Pathol 2010;34:243-255.

4 Sica G, Yoshizawa A, Sima CS, et al. A grading system of lung adenocarcinomas based on histologic pattern is predictive of disease recurrence in stage I tumors. Am J Surg Pathol 2010;34:1155-1162.

5 Barletta JA, Yeap BY, Chirieac LR. Prognostic significance of grading in lung adenocarcinoma. Cancer 2010;116:659-669.

6 Kurokawa T, Matsuno Y, Noguchi M, et al. Surgically curable "early" adenocarcinoma in the periphery of the lung. Am J Surg Pathol 1994;18:431-438.

7 Asamura H, Ando M, Matsuno Y, et al. Histopathologic prognostic factors in resected adenocarcinomas: is nuclear DNA content prognostic? Chest 1999;115:1018-1024.

8 Petersen I, Kotb WF, Friedrich KH, et al. Core classification of lung cancer: correlating nuclear size and mitoses with ploidy and clinicopathological parameters. Lung Cancer 2009;65:312-318.

9 Nakazato Y, Minami Y, Kobayashi H, et al. Nuclear grading of primary pulmonary adenocarcinomas: correlation between nuclear size and prognosis. Cancer 2010;116:2011-2019.

10 Kobayashi Y, Yokose T, Kawamura K, et al. Cytologic factors associated with prognosis in patients with peripheral adenocarcinoma of the lung measuring $3 \mathrm{~cm}$ or less in greatest dimension. Cancer 2005;105:44-51.

11 Travis WD, Brambilla E, Noguchi M, et al. International association for the study of lung cancer/American thoracic society/European respiratory society international multidisciplinary classification of lung adenocarcinoma. J Thorac Oncol 2011;6:244-285.

12 Yoshizawa A, Motoi N, Riely GJ, et al. Impact of proposed IASLC/ATS/ERS classification of lung ade- nocarcinoma: prognostic subgroups and implications for further revision of staging based on analysis of 514 stage I cases. Mod Pathol 2011;24:653-664.

13 Yoshizawa A, Sumiyoshi S, Moreira AL, et al. Validation of the IASLC/ATS/ERS lung adenocarcinoma classification and use of comprehensive histologic subtyping for architectural grading in 432 Japanese patients. Mod Pathol 2011;24:429A.

14 Meyer JS, Alvarez C, Milikowski C, et al. Breast carcinoma malignancy grading by Bloom-Richardson system vs proliferation index: reproducibility of grade and advantages of proliferation index. Mod Pathol 2005;18:1067-1078.

15 Thomas JS, Kerr GR, Jack WJ, et al. Histological grading of invasive breast carcinoma - a simplification of existing methods in a large conservation series with long-term follow-up. Histopathology 2009;55: 724-731.

16 Rioux-Leclercq N, Karakiewicz PI, Trinh QD, et al. Prognostic ability of simplified nuclear grading of renal cell carcinoma. Cancer 2007;109:868-874.

17 Epstein JI. The new World Health Organization/ International Society of Urological Pathology (WHO/ ISUP) classification for TA, T1 bladder tumors: is it an improvement? Crit Rev Oncol Hematol 2003;47:83-89.

18 Lipponen PK, Eskelinen MJ, Jauhiainen K, et al. Independent clinical, histological and quantitative prognostic factors in transitional-cell bladder tumours, with special reference to mitotic frequency. Int J Cancer 1992;51:396-403.

19 Edge SB, Byrd DR, Compton CC, et al. AJCC Cancer Staging Manual. 7th edn. Springer: New York, NY, 2009, pp 253-270.

20 van Diest PJ, van der Wall E, Baak JP. Prognostic value of proliferation in invasive breast cancer: a review. J Clin Pathol 2004;57:675-681.

21 Travis WD, Rush W, Flieder DB, et al. Survival analysis of 200 pulmonary neuroendocrine tumors with clarification of criteria for atypical carcinoid and its separation from typical carcinoid. Am J Surg Pathol 1998;22:934-944.

22 Baak JP. Mitosis counting in tumors. Hum Pathol 1990;21:683-685.

23 Maezawa N, Tsuta K, Shibuki Y, et al. Cytopathologic factors can predict invasion in small-sized peripheral lung adenocarcinoma with a bronchioloalveolar carcinoma component. Cancer 2006;108:488-493.

24 Cerruto CA, Brun EA, Chang D, et al. Prognostic significance of histomorphologic parameters in diffuse malignant peritoneal mesothelioma. Arch Pathol Lab Med 2006;130:1654-1661.

25 Thompson LD. Pheochromocytoma of the Adrenal gland Scaled Score (PASS) to separate benign from malignant neoplasms: a clinicopathologic and immunophenotypic study of 100 cases. Am J Surg Pathol 2002;26:551-566.

26 Dong B, Sato M, Sakurada A, et al. Computed tomographic images reflect the biologic behavior of small lung adenocarcinoma: they correlate with cell proliferation, microvascularization, cell adhesion, degradation of extracellular matrix, and K-ras mutation. J Thorac Cardiovasc Surg 2005;130:733-739.

27 Guillou L, Coindre JM, Bonichon F, et al. Comparative study of the National Cancer Institute and French Federation of Cancer Centers Sarcoma Group grading systems in a population of 410 adult patients with soft tissue sarcoma. J Clin Oncol 1997;15:350-362. 
28 Hasegawa T, Yokoyama R, Matsuno Y, et al. Prognostic significance of histologic grade and nuclear expression of beta-catenin in synovial sarcoma. Hum Pathol 2001;32:257-263.

29 Poleri C, Morero JL, Nieva B, et al. Risk of recurrence in patients with surgically resected stage I non-small cell lung carcinoma: histopathologic and immunohistochemical analysis. Chest 2003;123:1858-1867.

30 Demarchi LM, Reis MM, Palomino SA, et al. Prognostic values of stromal proportion and PCNA, Ki-67, and p53 proteins in patients with resected adenocarcinoma of the lung. Mod Pathol 2000;13:511-520.

31 Kalogeraki A, Tzardi M, Zoras O, et al. Apoptosis and cell proliferation correlated with tumor grade in patients with lung adenocarcinoma. In Vivo 2010; 24:667-670.

32 Terasaki H, Niki T, Matsuno Y, et al. Lung adenocarcinoma with mixed bronchioloalveolar and invasive components: clinicopathological features, subclassification by extent of invasive foci, and immunohistochemical characterization. Am J Surg Pathol 2003; 27:937-951.

33 Yang Q, Mori I, Sakurai T, et al. Correlation between nuclear grade and biological prognostic variables in invasive breast cancer. Breast Cancer 2001;8:105-110.

34 Cross SS. Grading and scoring in histopathology. Histopathology 1998;33:99-106.
35 Barry M, Sinha SK, Leader MB, et al. Poor agreement in recognition of abnormal mitoses: requirement for standardized and robust definitions. Histopathology 2001;38:68-72.

36 Ikeguchi M, Sakatani T, Endo K, et al. Computerized nuclear morphometry is a useful technique for evaluating the high metastatic potential of colorectal adenocarcinoma. Cancer 1999;86:1944-1951.

37 Subramanian J, Simon R. Gene expression-based prognostic signatures in lung cancer: ready for clinical use? J Natl Cancer Inst 2010;102:464-474.

38 Bryant CM, Albertus DL, Kim S, et al. Clinically relevant characterization of lung adenocarcinoma subtypes based on cellular pathways: an international validation study. PLoS One 2010;5:e11712.

39 Felip E, Rosell R, Maestre JA, et al. Preoperative chemotherapy plus surgery versus surgery plus adjuvant chemotherapy versus surgery alone in early-stage non-small-cell lung cancer. J Clin Oncol 2010;28: 3138-3145.

40 Strauss GM, Herndon II JE, Maddaus MA, et al. Adjuvant paclitaxel plus carboplatin compared with observation in stage IB non-small-cell lung cancer: CALGB 9633 with the Cancer and Leukemia Group B, Radiation Therapy Oncology Group, and North Central Cancer Treatment Group Study Groups. J Clin Oncol 2008;26:5043-5051. 